\title{
Simplifying Medication Regimens for People Receiving Community-Based Home Care Services: Outcomes of a Non-Randomized Pilot and Feasibility Study
}

This article was published in the following Dove Press journal: Clinical Interventions in Aging

\author{
Janet K Sluggett $\mathbb{D}^{1-3}$ \\ Choon Ean Ooi \\ Stephanie Gibson (ID ${ }^{1}$ \\ Manya T Angley $\mathbb{D}^{4}$ \\ Megan Corlis $\mathbb{1 D}^{3,5}$ \\ Michelle E Hogan ${ }^{3,5}$ \\ Tessa Caporale ${ }^{5}$ \\ Georgina A Hughes ${ }^{1,4}$ \\ Jan Van Emden ${ }^{3,5}$ \\ J Simon Bell ${ }^{1,3,6}$
}

'Centre for Medicine Use and Safety, Faculty of Pharmacy and Pharmaceutical Sciences, Monash University, Parkville, Victoria, Australia; ${ }^{2}$ University of South Australia, UniSA Allied Health and Human Performance, Adelaide, South Australia, Australia; ${ }^{3}$ National Health and Medical Research Council (NHMRC) Cognitive Decline Partnership Centre, Hornsby Ku-ring-gai Hospital, Hornsby, New South Wales, Australia; ${ }^{4}$ University of South Australia, UniSA Clinical and Health Sciences, Adelaide, South Australia, Australia; ${ }^{5} \mathrm{Helping} \mathrm{Hand}$ Aged Care, North Adelaide, South Australia, Australia; ${ }^{6}$ Department of Epidemiology and Preventive Medicine, School of Public Health and Preventive Medicine, Monash University, Melbourne, Victoria, Australia

Correspondence: Janet K Sluggett Email janet.sluggett@unisa.edu.au
Purpose: Being able to manage a complex medication regimen is key to older people continuing to live at home. This study determined the feasibility of a multi-component intervention to simplify medication regimens for people receiving community-based home care services.

Patients and Methods: Research nurses recruited people receiving community-based home care services to participate in this non-randomized pilot and feasibility study (Australian New Zealand Clinical Trials Registry ACTRN12618001130257). Participants received a one-off clinical pharmacist intervention comprising medication reconciliation, assessment of capacity to self-manage medications, and application of a structured 5-step tool to identify medication simplification opportunities. A mixed-methods feasibility assessment with an explanatory design was undertaken to assess recruitment, protocol adherence and stakeholder acceptability. Data from interviews with 12 stakeholders were thematically analyzed. Secondary outcome measures, including medication discrepancies, and changes in number of medication administration times per day, quality of life, medication adherence and health service utilization, were determined over a 4-month follow-up.

Results: Twenty-five out of the target 50 participants were recruited. Initial recruitment was impacted by apparent uncertain role responsibilities in medication management, with some clients who declined to participate perceiving they would be unlikely to benefit or being reluctant to change regimens. However, with few exceptions, participants who received intervention did so with a high degree of protocol adherence and acceptability. Stakeholders valued the intervention and supported wider implementation. Discrepancies between the baseline medication history from the general medical practitioner and the pharmacist-compiled "best possible medication history" were identified for all participants' regimens (median of 6 per participant), with one-third resolved at follow-up. Simplification was possible for 14 participants $(56 \%)$ and implemented for $7(50 \%)$ at follow-up. No significant changes in other secondary outcomes were observed.

Conclusion: The intervention was delivered as planned, and valued by stakeholders. Recruitment barriers should be addressed before wider implementation.

Keywords: aged care, medication management, community services, medication simplification, Australia

\section{Introduction}

Older people often have high rates of multimorbidity and polypharmacy resulting in complex medication regimens. In the United States (US), the prevalence of polypharmacy (use of five or more medications) in people aged $\geq 65$ years increased from $13 \%$ to $39 \%$ between 1990 and $2010 .{ }^{1}$ Polypharmacy is experienced by $38 \%$ 
of older Australian men. ${ }^{2}$ Managing multiple medications can be complex, time-consuming and stressful for older people and caregivers. $^{3-6}$ Impaired cognition, dexterity, mobility, hearing and/or vision, and frailty contribute to reduced ability to manage a complex regimen., ${ }^{4,8}$ Complexity arises due to multiple formulations (eg, inhalers, patches), a greater number of administration times and special administration instructions (eg, crushing). ${ }^{3}$ Having a complex medication regimen may contribute to non-adherence, ${ }^{9}$ hospitalizations, ${ }^{10}$ medication errors and adverse drug events. ${ }^{11}$

Being able to manage a complex medication regimen is key to older people continuing to live at home. ${ }^{4,12}$ Older people often receive support from family members and/or community-based care packages to manage medications. ${ }^{4}$ Over $60 \%$ of Australians receiving an aged care assessment have $\geq 1$ limitation in instrumental activities of daily living (ADLs), including difficulty managing medications. ${ }^{13}$ A US survey of informal caregivers reported that $80 \%$ assisted with obtaining medications, 54\% with medication schedule planning and $35 \%$ with medication administration for older people. $^{14}$

Community-dwelling older people are at high risk of medication-related harm. Self-reported medication administration error rates of $12-59 \%$ have been reported. ${ }^{15}$ In the US and Canada, adverse events are experienced by $13 \%$ of home care services recipients. ${ }^{16}$ A retrospective review of 100 Australians receiving home nursing care found $41 \%$ had a medication error, $13 \%$ required medical attention and $9 \%$ were hospitalized due to an adverse drug event. $^{17}$

Medication regimen simplification is one promising strategy to increase the capacity of older people to selfmanage medications. It involves reducing the number of administration times per day through strategies such as administering multiple medications at the same time and using longer-acting formulations and combination formulations, without changing therapeutic intent. ${ }^{18}$ An electronic simplification application trialled in 1500 US community-dwelling older people found $41 \%$ of regimens could be simplified. ${ }^{19}$ Simplification strategies applied within an outpatient cardiovascular cohort reduced the number of administration times and improved selfreported adherence. ${ }^{20}$

The Medication Regimen Simplification Guide for Residential Aged CarE (MRS GRACE) was developed, validated and applied in a clinical trial in residential aged care facilities (RACFs). ${ }^{18,21-23}$ The aim of this study was to determine the feasibility of a multicomponent intervention to simplify medication regimens for people receiving community-based home care services. Quantitative and qualitative evaluation were undertaken to determine feasibility of a future randomized controlled trial (RCT). The full list of primary and secondary study objectives are outlined in the published study protocol. ${ }^{24}$

\section{Patients and Methods Design, Setting and Participants}

This was a non-randomized pilot and feasibility study and all participants received the intervention. The study was prospectively registered with the Australian New Zealand Clinical Trials Registry (ACTRN12618001130257) and the protocol was published. ${ }^{24}$

Participants were older recipients of home care services delivered through government-funded packages that subsidize providers to deliver services that meet an individual's care needs. ${ }^{25,26}$ The home care services included Commonwealth Home Support Programme services or Home Care Packages. ${ }^{24}$ The participants resided in South Australia, the fourth largest state in Australia, and received services from a private not-for-profit aged care provider organization. People that were non-English speaking, had less than two regular administration times, were estimated to have $<3$ months to live, or were unwell during recruitment were excluded. There were no restrictions relating to participant age, clinical profile or presence of informal caregivers.

\section{Recruitment}

Research nurses were contracted by the community-based home care provider organization to recruit participants for the intervention. The research nurses had good knowledge of organizational processes, policies and procedures but did not provide direct care for potential participants. Research nurses initially invited all eligible individuals with higher care needs and receiving formal medication administration assistance from one South Australian community-based home care provider. Potential participants were contacted by mail and the research nurses then conducted follow-up telephone calls to provide verbal information about the study and seek written informed consent to participate. Due to a high rate of refusals to participate, research nurses then invited all individuals with funding packages indicative of higher care needs (ie, level 3 or 4 packages) who did not receive medication administration 
assistance. To further maximize recruitment, research nurses then identified all remaining people receiving community-based home care services, randomly selected up to 10 individuals with a surname starting with each letter of the alphabet (where possible), and invited them to participate. A research nurse and clinical pharmacist also attended community events run by the organization and allied health professionals employed by the organization were provided with information sheets for dissemination.

\section{Data Collection}

Data were collected at baseline and 4-months post-study entry by research nurses. Research nurses spoke with the participant and/or informal caregivers, and liaised with the participant's community pharmacy, general medical practitioner (GP) and the community-based home care provider to obtain demographic, medical and medication history information. The following questionnaire data were collected via participant self-report and/or interviews with caregivers who had known the participant for $>2$ weeks: Dementia severity, determined using the 12-item Dementia Severity Rating Scale (DSRS); ${ }^{27}$ activities of daily living (ADL) assessed using the 6-item Katz scale; ${ }^{28}$ and frailty, assessed using the 5 -item FRAIL screening test scale. ${ }^{29}$

\section{Intervention}

A clinical pharmacist delivered the intervention to all participants after baseline data collection. It involved 3 components: medication reconciliation, validated assessment of capacity to self-manage medications, and identification of medication simplification opportunities using a structured 5-step process. ${ }^{24}$ Medication reconciliation was performed to ensure any simplification recommendations were based on an accurate medication list. After compiling a preliminary medication history from baseline data collected by the research nurse as described above, the pharmacist interviewed the participant in their own home to confirm current medication use and compiled a "best possible medication history" (BPMH). The pharmacist assessed the participant's ability to self manage their medications using the Drug Regimen Unassisted Grading Scale (DRUGS) ${ }^{30}$ and identified simplification opportunities using a version of MRS GRACE that was modified for use among recipients of community-based home care services. ${ }^{18,24}$ As part of applying MRS GRACE, the participant's willingness to have their medication regimen simplified was discussed.

The pharmacist prepared a written report that included the reconciled BPMH, medication discrepancies between the
BPMH and the baseline information provided by the GP, a "medication management statement" discussing adherence and capacity to self-manage, and simplification recommendations. Findings were discussed with a senior registered nurse at the community-based home care provider organization and the report was forwarded to the GP. The clinical pharmacist liaised with the participant's GP and community pharmacist as needed to assist implementation of medication management recommendations. Each participant was offered a copy of the report and an updated medication list.

\section{Primary Outcome}

The primary outcome was a statement of study feasibility that considered (a) recruitment and retention, (b) protocol adherence and (c) stakeholder acceptability of the recruitment processes, assessments, intervention delivery and secondary outcome measures. Feasibility was assessed using a mixed-methods approach with an explanatory design that included document analysis, and focus groups/semi-structured interviews.

\section{Document Analysis}

Eligibility, recruitment and retention rates were assessed via document analysis of field notes, recruitment logs, emails, the clinical pharmacist's reports and meeting minutes. Uptake of simplification recommendations was assessed by the research team from medication data at follow-up.

\section{Qualitative Approach}

Face-to-face semi-structured interviews undertaken postintervention explored protocol adherence and adaptations; acceptability of recruitment processes, questionnaires, intervention delivery and secondary outcome measures; and barriers and enablers to recruitment, intervention delivery and future implementation.

To determine potential interview participants, the research team asked the research nurse and clinical pharmacist to nominate GPs they had liaised with as part of the project. The research nurse and pharmacist also nominated participants receiving home care services who could be suitable to approach with a request for interview. The deidentified study identification numbers for names of 20 participants provided by the research nurse or pharmacist were categorized according to whether simplification was possible, and if so, whether simplification recommendations were implemented. From this list, six individuals were randomly selected to ensure the sample included a mix of 
participants with different simplification outcomes. The aged care provider organization nominated representatives to be approached for interview. Overall, 14 individuals from all stakeholder groups, including community-based home care provider research and development (R\&D) representatives $(n=2)$, GPs $(n=2)$, clinical pharmacist, research nurse, community-based registered nurse, participants $(n=6)$ and an informal caregiver were invited to participate.

Interviews were conducted face-to-face by two researchers (JKS and GH) who had pharmacy backgrounds. The interview guide was adapted from a guide the two researchers had previously pilot tested for face validity and used to conduct stakeholder interviews for a mixed-methods process evaluation of an RACF-based medication simplification intervention. ${ }^{21}$ Study participants were interviewed in their usual place of residence. Other interviews were conducted in the participant's usual place of work in a private office. The interviews were audiotaped and transcribed verbatim by an external transcription service. Brief notes were taken during the interview as necessary and no repeat interviews were conducted.

The interviews were analyzed in accordance with the reflexive thematic analysis approach outlined by Braun and Clarke. ${ }^{31}$ This involved repeated reading of the transcripts, coding, collating into themes, and reviewing and naming themes. The main codes were predominantly determined using existing topic knowledge (ie, a deductive approach). Interview data were coded by one investigator $(\mathrm{GH})$ and then reviewed by two other investigators (CEO, JKS). After writing the results, quotations that illustrated key themes were identified. Analysis was conducted using an iterative process that commenced after the first interview and continued throughout the interview process to inform subsequent data capture.

\section{Secondary Outcomes}

Secondary outcomes were the total number of administration times over 24-hours for regular medications at 4-months compared with baseline. The number of administration times incorporated prescription, non-prescription, and complementary and alternative medications administered at least once per day on a regular basis, irrespective of dosage form, in accordance with previous related studies. $^{22,23}$ Nutritional drinks and medications administered "when required", short term, or less frequently than daily were excluded when calculating the number of administration times.
Other secondary measures included: the total number of individual regular medication doses administered over a 24-hour period, medication adherence assessed using the Self-Efficacy for Appropriate Medication use Scale (SEAMS), ${ }^{32}$ quality of life using the Quality of Life in Alzheimer's Disease scale (QoL-AD), ${ }^{33}$ participant satisfaction using the Short Assessment of Patient Satisfaction (SAPS), ${ }^{34}$ provision a Home Medicines Review (a government-subsidized, comprehensive medicines review provided in the person's home by an accredited pharmacist in conjunction with the individual's usual GP), falls, ambulance call-outs, emergency department (ED) visits, hospital admissions, RACF admissions and all-cause mortality. Types of simplification recommendations and time spent delivering the intervention were also assessed. Data on possible medication incidents were not collected because only one participant received medication management assistance from the community-based home care provider. Data self-report and completion rates were reviewed to assess feasibility of data collection and secondary outcomes. Secondary outcomes were assessed using descriptive statistics and changes at follow-up were analyzed using the paired $t$-test or Wilcoxon signed rank test (continuous variables), or McNemar's test (categorical variables).

The baseline medication history provided by each participant's GP was compared to the BPMH prepared by the clinical pharmacist to identify discrepancies. Two pharmacist members of the research team (SG, CEO) independently reviewed and categorized the clinical significance of each discrepancy using a validated tool and assessed the likelihood of an adverse outcome should the discrepancy not be corrected. ${ }^{35,36}$ Adverse outcomes were defined as deterioration in symptom control, exacerbation of medical conditions and adverse drug events.

\section{Ethical Considerations}

Ethical approval was obtained from the Monash University Human Research Ethics Committee (ID 11614) and the Helping Hand ethical review panel. The study was conducted in accordance with the Declaration of Helsinki. Weekly meetings were held to monitor recruitment, data collection and intervention delivery.

Written informed consent to participate in the intervention was obtained directly from an individual where possible. Consent was sought from the person's guardian, next of kin or significant other when the person was unable to provide written informed consent to participate. Written 
informed consent was obtained from each interview participant prior to interview commencement.

\section{Results}

\section{Participant Characteristics}

Recruitment for the intervention took place between 16 July and 16 October 2018, with the first participant enrolled on 22 August. The characteristics of the 25 individuals who received the intervention are described in Table 1.

Nine semi-structured interviews were conducted with 12 participants (a research nurse, a community nurse, two aged care provider $R \& D$ representatives, the clinical pharmacist, six study participants who received the intervention and one informal caregiver) between December 2018 and January 2019.

\section{Primary Outcome: Study Feasibility} Recruitment and retention

Overall, 262 individuals were screened for eligibility, 222 were invited and 27 agreed to participate (Figure 1). Of these, 25 met the inclusion criteria, provided written consent and received the intervention. The recruitment target was revised down from 50 to 25 participants due to the recruitment challenges that were encountered.

Of the 222 individuals invited, 120 (54\%) formally declined to participate (Figure 1). Reasons for declining were provided by 83 individuals and included perception of no benefit (43\%), unwilling to change medication regimen $(17 \%)$, and too busy (12\%). One participant who received the intervention withdrew from the study, for reasons unrelated to the intervention, before follow-up (96\% retention rate).

Key barriers and enablers to recruitment were further explored during the stakeholder interviews. There was general consensus that understanding the importance of research and broader societal benefits facilitated participation. It was suggested that promotion through general practices and community-based home care provider organizations, and via email and/or text messages could increase recruitment rates.

Complexity of the information sheet, older age and trust in an individual's usual GP and community pharmacist were perceived as potential barriers to participation.

I think some people say, “I'm too old to do research. I'm too old," and I said, "Oh no, you're never too old. We can use everybody's information." But the main was, yeah, that just it was working, "My doctor looks after it. It's all packed in the blister pack. I don't have to do anything. It's all there. I don't need to touch it." [Research nurse]
Table I Baseline Characteristics of Study Participants

\begin{tabular}{|c|c|}
\hline & $\begin{array}{l}\text { Participants } \\
\mathrm{n}=\mathbf{2 5}\end{array}$ \\
\hline Age (years) at study entry (mean, $\pm S D$ ) & $79.0 \pm 7.5$ \\
\hline Male (n, \%) & $9(36 \%)$ \\
\hline Self-consent for study participation ( $\mathrm{n}, \%)$ & 21 (84\%) \\
\hline Living alone (n, \%) & $14(56 \%)$ \\
\hline $\begin{array}{l}\text { Type of community-based home care service } \\
\text { received }(n, \%)\end{array}$ & \\
\hline $\begin{array}{l}\text { Commonwealth Home Support Programme } \\
\text { services }\end{array}$ & $15(60 \%)$ \\
\hline Home Care Packages & $10(40 \%)$ \\
\hline Medical conditions $(\mathrm{n}, \%)$ & \\
\hline Hypertension & 18 (72\%) \\
\hline Hyperlipidemia & $16(64 \%)$ \\
\hline Osteoarthritis & $16(64 \%)$ \\
\hline Ischemic heart disease & $9(36 \%)$ \\
\hline Type 2 diabetes & $9(36 \%)$ \\
\hline Depression & $9(36 \%)$ \\
\hline History of fracture & $9(36 \%)$ \\
\hline Cerebrovascular disease & $8(32 \%)$ \\
\hline Dementia & $3(12 \%)$ \\
\hline FRAIL score ${ }^{\mathrm{a}}$, median (IQR) & $2(I-3)$ \\
\hline $\begin{array}{l}\text { Katz Activities of Daily Living scale score, median } \\
\text { (IQR) }\end{array}$ & $5(4-6)$ \\
\hline $\begin{array}{l}\text { Number of administration times for medications } \\
\text { taken regularly }(n, \%)\end{array}$ & \\
\hline Twice daily & $13(52 \%)$ \\
\hline Three times daily & $6(24 \%)$ \\
\hline Four or more times daily & $6(24 \%)$ \\
\hline Total number of medications (mean, $\pm \mathrm{SD}$ ) & $13.8 \pm 3.9$ \\
\hline Medication administration ( $\mathrm{n}, \%)$ & \\
\hline Self-administration & 17 (68\%) \\
\hline $\begin{array}{l}\text { Receives assistance through community-based } \\
\text { home care provider }\end{array}$ & I (4\%) \\
\hline Receives assistance from a family member & $7(28 \%)$ \\
\hline $\begin{array}{l}\text { Drug Regimen Unassisted Grading Scale (DRUGS) } \\
\text { score, median (IQR) }\end{array}$ & \\
\hline All participants & $100(53.8-100)$ \\
\hline $\begin{array}{l}\text { Participants who were self-administering } \\
\text { medications }(n=17)\end{array}$ & $100(67.1-100)$ \\
\hline
\end{tabular}

Note: ${ }^{a} n=20$ participants (weight component of FRAlL score missing for 5 participants).

Abbreviations: DRUGS, Drug Regimen Unassisted Grading Scale; FRAIL, Fatigue, Resistance, Ambulation, Illnesses, and Loss of Weight; IQR, interquartile range; SD, standard deviation.

Stakeholders observed resistance to change in some individuals that potentially impacted the decision to participate and uptake of simplification recommendations. Interviewees speculated this may be associated with fear 


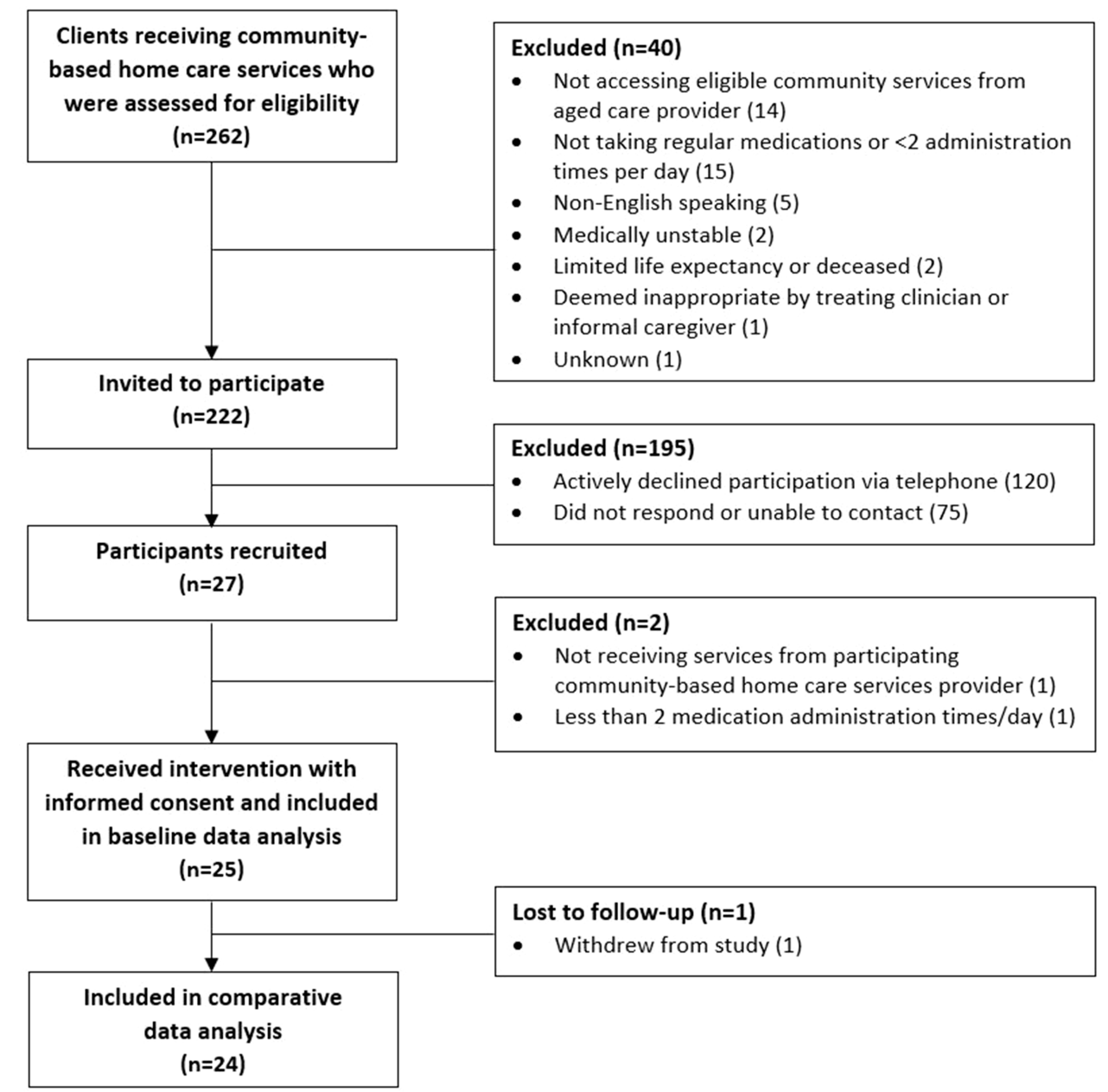

Figure I Participant flow chart.

of losing control of routine and autonomy in medication management.

... But I think there's a lot to say for someone sticking to a routine, having control over it and then when there's change, and it sort of messes it all up, they see it as it being messed up a little bit, and I wonder if, I think that's a difficulty. [Research nurse]

\section{Protocol Adherence and Adaptations}

The document analysis and description provided by the pharmacist during the interview indicated the intervention was delivered in accordance with the protocol. All 25 participants received all three intervention components. The clinical pharmacist spent a median of 70 minutes with the participant in their home, with most of that time spent determining the BPMH for that individual (Table 2).

The DRUGS assessment showed most participants were able to self-manage their medications (Table 1).
The five participants who were self-administering medications that did not achieve the maximum DRUGS score used a dose administration aid prepared by their

Table 2 Time Spent by the Clinical Pharmacist on Intervention Delivery and Follow-Up

\begin{tabular}{|l|l|}
\hline Intervention Components & $\begin{array}{l}\text { Time Spent by the } \\
\text { Pharmacist (mins), Median } \\
\text { (IQR) }\end{array}$ \\
\hline $\begin{array}{l}\text { Total time spent for home visit } \\
\text { Medication reconciliation } \\
\text { Administration of the Drug } \\
\text { Regimen Unassisted Grading Scale } \\
\text { Identifying opportunities for } \\
\text { medication simplification }\end{array}$ & $\begin{array}{l}55(65-75) \\
15(10-15)\end{array}$ \\
\hline $\begin{array}{l}\text { Total time spent preparing report } \\
\text { Total time spent communicating } \\
\text { findings verbally with stakeholders }\end{array}$ & $60(60-70)$ \\
$10(5-15)$
\end{tabular}

Abbreviation: IQR, interquartile range. 
community pharmacist. These individuals could not always identify individual medications, or indications for use, but could administer them accurately.

The clinical pharmacist identified opportunities to simplify medication regimens for 14 (56\%) participants. Recommendations for change in administration time were made for 13 participants and mostly involved changing administration times for oral medication(s). An alternative, therapeutic equivalent medication that offered a less frequent dosing schedule was recommended for one participant. No recommendations were made to change to modified-release formulations or combination products.

The clinical pharmacist felt positive about the structure, intent and potential value of the intervention.

The tool [revised MRS GRACE] is a great sort of starting point, and a great way to set the scene ... I draw a lot on my professional experience, but I did revisit the tool, just to think ' $\mathrm{OK}$, am I on task, am I doing things according to the process, the protocol. I did revisit it. [Clinical pharmacist]

However, the clinical pharmacist described experiencing difficulty in separating the intervention from a comprehensive medication review service. The clinical pharmacist often felt compelled to resolve medication-related problems and provide education, thereby delivering a more thorough service than originally intended. Health professionals perceived a lack of complete information and infrastructure to facilitate timely communication and handover to other health professionals as a potential barrier to intervention delivery and implementation of recommendations.

So, I was feeling quite deprived by not having full access to discharge summaries and that data ... I still think I was able to deliver a good service. And that's where your home care pharmacist would then sort of handover to the [general] practice pharmacist, I think. And I guess ultimately you would want your home care database talking to the GP software, ideally. [Clinical pharmacist]

\section{Acceptability of recruitment processes, questionnaires, intervention delivery and secondary outcome measures}

Interviewees valued the concept of medication simplification and possible broader benefits. The research nurse made an important contribution to facilitating study conduct and streamlined data collection at baseline and 4-month followup. Some questionnaire items were perceived as difficult to answer, confusing or repetitive, although interactions with the study team were valued. Participants generally perceived the recruitment process and intervention delivery methods positively and delivery in participants' homes was perceived as advantageous and enabled collection of comprehensive health-related information.

Most people want a simpler life. They want their life, things to be streamlined ... It [medication management] takes up a big part of their day-to-day responsibilities and self-care. [Clinical pharmacist]

Examination of medication data collected at follow-up showed recommendation(s) to alter administration time(s) in 7 out of 13 participants were implemented at follow-up, resulting in a 54\% implementation rate. Interview findings suggest that participant's trust in the pharmacist's advice, simplicity of the recommendations and subsequent discussions with their treating GP encouraged implementation. Study participants often appreciated the recommendations and education provided by the clinical pharmacist and some reported benefits following simplification.

It's just that mine were daily tablets and it was only a couple of things that I took at night. [The clinical pharmacist] said 'No, take them in the day time.' So, I was quite happy to do that. [Study participant 3]

... One client on my visits were absolutely so happy with one of the recommendations that [the clinical pharmacist] had made, and that was around a Parkinson's medication and being able to open the capsule. They thought it was ... longacting and that you couldn't actually open the capsule, and now they can. They had actually been looking at getting a pump, and they told the specialist that they didn't need it anymore because the service had said this, they didn't need it, and so that was put off, that's been actually put off. That's what they told me in the interview. So that is fantastic. [Research nurse]

While valuing the intervention, some participants chose not to implement the simplification strategies suggested by the pharmacist. Barriers to implementation included resistance to change medications packed in dose administration aids, fear of not adhering to a revised routine, and perceived low burden of current regimen or limited value of the suggested change.

[Clinical pharmacist's name] came and asked us if we would change to take the medication from the morning to the evening, and I thought that's going to be a bit confusing. Then I have to take medication ... before the 
meal. When I'm cooking, I will have forgotten to take it so then I have to wait two hours after to take it. So, I thought it is better to stay with the morning program. [Informal caregiver, explaining why the simplification recommendation was not implemented]

After the intervention, all participants were provided with a medication list, informed by the BPMH, to aid medication management. Stakeholders generally perceived this an important component of the intervention.

And I think that closing that loop of giving the participant a copy of the report or the reconciliation after was really important as well as some of those that requested it. I think the process was good. [Community-based home care provider $\mathrm{R} \& \mathrm{D}$ representative 1]

Overall, interviewees generally perceived the intervention as valuable and suitable for broader implementation. Pharmacists were viewed as best placed to deliver the intervention in everyday practice, in collaboration with GPs and community-based home care providers. Use of a shared health record was suggested by the clinical pharmacist to facilitate communication and continuity of care in future interventions. Routine provision of a medication management service that includes medication simplification was considered important when an individual first accesses an aged care service, with the frequency of subsequent medication simplification activities suggested as three-monthly to two-yearly thereafter depending on individuals' needs. It was also suggested the intervention could be provided following a hospitalisation as medication regimens may be different on discharge. Stakeholders perceived the intervention could be incorporated into existing comprehensive medication review services that are currently available upon
GP referral in Australia, or alternatively, integrated into home care packages.

\section{Secondary Outcomes Data Acquisition and Questionnaire Completion Rates}

Research nurses obtained a medical history from the participant's GP and a medication history from the GP and usual community pharmacy for $83 \%$ to $96 \%$ of participants (Supplementary Table 1). Medical history was frequently recorded by the community-based home care provider organization, but was not always consistent with the information provided by the GP. Conversely, a current medication list was rarely available from the community-based home care provider organization as this information is only captured for individuals who have medications administered by community-based home care nurses.

The proportion of participants able to self-report information for the SEAMS, FRAIL, QoL-AD and SAPS questionnaires at baseline and follow-up ranged from $71 \%$ to 96\% (Supplementary Table 2). All elements of the SEAMS, QoL-AD, Katz ADL and SAPS questionnaires were completed for most participants ( $92 \%$ to $100 \%)$, but completion of the FRAIL was lower (79\% and $63 \%$ ) as weight information was not always provided. Completion rates for the DSRS were low because the research nurse felt uncomfortable asking participants certain questions, and therefore only administered the questionnaire in a small number of cases when a caregiver informant was available (Supplementary Table 3).

\section{Medication Discrepancies}

At least one discrepancy between the GP current medication list provided at baseline and the BPMH was observed

Table 3 Nature and Number of Discrepancies Observed with the Medication List Collected at Baseline from the Participant's General Medical Practitioner Compared to the Best Possible Medication History ( $n=24$ Participants) ${ }^{a}$

\begin{tabular}{|c|c|c|c|}
\hline Discrepancy Type & $\begin{array}{l}\text { No. of Participants with This } \\
\text { Type of Discrepancy (n, \%) }\end{array}$ & $\begin{array}{l}\text { Total no. of Discrepancies } \\
\text { Among all Participants }\end{array}$ & $\begin{array}{l}\text { Proportion of Total } \\
\text { Discrepancies (\%) }\end{array}$ \\
\hline Omission ${ }^{\mathrm{b}}$ & $24(100 \%)$ & 70 & $41.7 \%$ \\
\hline Addition $^{c}$ & $20(83 \%)$ & 43 & $25.6 \%$ \\
\hline Dose, frequency or time of administration & $20(83 \%)$ & 40 & $23.8 \%$ \\
\hline Route or formulation & $6(25 \%)$ & 7 & $4.2 \%$ \\
\hline Generic duplication ${ }^{\mathrm{d}}$ or therapeutic substitution & $5(21 \%)$ & 7 & $4.2 \%$ \\
\hline Length of treatment & I (4\%) & 1 & $0.6 \%$ \\
\hline
\end{tabular}

Notes: ${ }^{\mathrm{G}} \mathrm{GP}$ list at baseline not available for one participant. ${ }^{\mathrm{b}} \mathrm{Client}$ taking a medication that is not on the GP medication list. ${ }^{\mathrm{c}} \mathrm{GP}$ medication list contains a medication that the client is not taking. ${ }^{\mathrm{G}} \mathrm{GP}$ medication list contains multiple(s) of the same medication and strength presented as different brand or generic names.

Abbreviation: GP, general medical practitioner. 
Table 4 Clinical Significance of Medication Discrepancies Assessed Independently by Two Pharmacists

\begin{tabular}{|c|c|c|c|}
\hline $\begin{array}{l}\text { Significance of } \\
\text { Medication } \\
\text { Discrepancy }\end{array}$ & $\begin{array}{l}\text { No. of Discrepancies } \\
\text { Observed at Baseline (n, \%) }\end{array}$ & $\begin{array}{l}\text { Resolved at } \\
\text { Follow-Up } \\
(n, \%)^{a}\end{array}$ & Examples \\
\hline $\begin{array}{l}\text { Unlikely to result in an } \\
\text { adverse outcome }\end{array}$ & $93(55.4)$ & $19 / 87$ (21.8\%) & $\begin{array}{l}\text { - Atorvastatin - GP directions are I tablet at night, partici- } \\
\text { - } \text { Lant takes I in the morning } \\
\text { particating eye drops - GP list states three times daily, } \\
\text { - Metformin extended release - GP list states Ig twice daily, } \\
\text { participant takes } 2 \mathrm{~g} \text { daily }\end{array}$ \\
\hline $\begin{array}{l}\text { Possibly will result in an } \\
\text { adverse outcome }\end{array}$ & $39(23.2)$ & I I/27 (40.7\%) & $\begin{array}{l}\text { - Empagliflozin on GP list, ceased during hospital admission } \\
\text { due to declining renal function } \\
\text { - Paracetamol sustained release not on GP list, participant } \\
\text { takes regularly twice daily for osteoarthritis pain } \\
\text { - Simvastatin listed twice on GP list as two different brand } \\
\text { names, may result in unintentional double dosing }\end{array}$ \\
\hline $\begin{array}{l}\text { Probably will result in an } \\
\text { adverse outcome }\end{array}$ & $36(21.4)$ & $18 / 30(60.0 \%)$ & $\begin{array}{l}\text { - Glimepiride on GP list, recently ceased due to } \\
\text { a hypoglycaemic episode while driving } \\
\text { - Short acting insulin not on GP list, participant receives } 3 \text { to } \\
10 \text { units with each meal } \\
\text { - Nebivolol not on GP list, previously commenced by con- } \\
\text { sultant physician }\end{array}$ \\
\hline TOTAL & $168(100)$ & 48// 44 (33.3\%) & \\
\hline
\end{tabular}

Note: a Data not available for all participants at follow-up.

Abbreviation: GP, general medical practitioner.

for all participants, with a median of six discrepancies per participant (interquartile range (IQR) 4.5-9) (Table 3). There were 168 discrepancies identified, with $21.4 \%$ classified as probable to cause an adverse outcome (Table 4). Inspection of GP medication lists provided showed onethird of the discrepancies were resolved at follow-up, with the higher-risk discrepancies more likely to be resolved (Table 4).

Interview participants identified there was further opportunity to review medical history, allergy and medication information for participants and resolve any discrepancies at follow-up.

... There was a difference between the blister pack and what the information that the pharmacist sent on the medication list, so the medication list for the pharmacy and the GP was the same but the blister pack was different. And so, I contacted the client, then I contacted the pharmacy ... [Research nurse]

\section{Changes in Secondary Outcomes at Follow-Up}

Comparison at baseline and follow-up showed no significant change in the number of medication administration times for regular medications over a 24-hour period, nor other secondary outcome measures (Table 5).

\section{Discussion}

This pilot and feasibility study showed value in a collaborative medication intervention among recipients of community-based home care services. The intervention was delivered according to the protocol and the acceptability among those who received the intervention was high. However, unexpected barriers to recruitment should be addressed before undertaking an RCT or implementing the service in routine clinical practice.

Considerable recruitment challenges were encountered, with only $12 \%$ of invitees agreeing to participate. However, this participation rate was comparable to $10-12 \%$ participation rates reported in studies of pharmacist medication management services for community-dwelling adults in Australia and the United Kingdom. ${ }^{37,38}$ Higher recruitment rates of $31-39 \%$ have been achieved when referrals are made by a person's usual pharmacist or GP. ${ }^{39,40}$ Process barriers to recruitment included the amount of recruitment 
Table 5 Comparison of Secondary Outcome Measures at Baseline and Four Months ( $\mathrm{n}=24$ Participants Unless Otherwise Stated)

\begin{tabular}{|c|c|c|c|}
\hline & Baseline & 4-Months & p-value \\
\hline Total no. of medication administration times in 24 hours for regular medications (median, IQR) & $2(2-3)$ & $2(2-3)$ & $0.092^{\mathrm{d}}$ \\
\hline Total no. of individual medication doses in 24 hours for regular medications (median, IQR) & $14(7.3-15.8)$ & $13.25(7.8-16.5)$ & $0.533^{\mathrm{d}}$ \\
\hline Self-Efficacy for Appropriate Medication use Scale (SEAMS) score (median, IQR) ${ }^{\mathrm{a}}$ & $38(32-39)$ & $39(37-39)$ & $0.145^{\mathrm{d}}$ \\
\hline Quality of Life in Alzheimer's Disease (QoL-AD) score (median, IQR) & $36(33.5-37.5)$ & $36.5(32-38.5)$ & $0.509^{\mathrm{d}}$ \\
\hline Short Assessment of Patient Satisfaction (SAPS) score (mean, SD) ${ }^{\mathrm{b}}$ & $22.5 \pm 3.0$ & $21.5 \pm 3.2$ & $0.278^{\mathrm{e}}$ \\
\hline Participant self-reported receiving a Home Medicines Review (n, \%) & $\mathrm{I}(4.2)^{\mathrm{c}}$ & $2(8.3)$ & $1.000^{f}$ \\
\hline Participant self-reported one or more falls ( $n, \%)$ & $7(29.2)^{c}$ & $5(20.8)$ & $0.480^{f}$ \\
\hline Participant self-reported one or more ambulance call outs with transportation $(n, \%)^{a}$ & $6(26.1)^{c}$ & $3(13.0)$ & $0.180^{f}$ \\
\hline Participant self-reported an emergency department visit (n, \%) & $5(20.8)^{c}$ & $\mathrm{I}(4.2 \%)$ & $0.103^{f}$ \\
\hline Participant self-reported a hospitalization ( $\mathrm{n}, \%)$ & $4(16.7)^{c}$ & $2(8.3)$ & $0.317^{f}$ \\
\hline Participant self-reported an emergency department visit or hospitalization (n, \%) & $8(33.3)^{c}$ & $3(12.5 \%)$ & $0.096^{f}$ \\
\hline Participant self-reported respite admission ( $\mathrm{n}, \%)$ & $0(0.0)^{c}$ & I (4.2\%) & $\mathrm{N} / \mathrm{A}$ \\
\hline
\end{tabular}

Notes: ${ }^{a} n=23$ participants. ${ }^{b} n=22$ participants. ${ }^{c}$ Outcomes experienced in the four months prior to study entry. ${ }^{d}$ Wilcoxon signed-rank test. ${ }^{e}$ Paired $t$-test. ${ }^{f} M c N e m a r ' s$ test. Abbreviations: IQR, interquartile range; N/A, not applicable; QoL-AD, Quality of Life in Alzheimer's Disease; SAPS, Short Assessment of Patient Satisfaction; SEAMS, SelfEfficacy for Appropriate Medication Use Scale.

information posted to individuals. Preparation of 1-page flyers describing the clinical rationale and potential benefits of participation for distribution via home-care nurses, at community groups, community pharmacies and/or at local general practices, and use of text or email approaches were suggested to increase reach. The community-based home care provider organization reported several administrative changes to the home care part of the organization immediately prior study commencement, including implementation of a new electronic administration system. This may facilitate improved identification of individuals most likely to benefit from the service in the future. Patient-centred barriers to recruitment included perception of lack of benefit and not wanting to change "current" medications. Similar barriers have been reported to the uptake of medication review services in Australia. ${ }^{41,42}$ Different perceptions regarding health professional's roles in medication management among individuals invited to participate may have contributed to these responses. For example, an individual who perceives their GP as responsible for medication management decisions might be less likely to participate in a medication simplification intervention delivered by their community-based aged care provider in conjunction with an external pharmacist. Trust in participants' usual GP was reported to influence participation, with greater willingness to participate if the intervention was seen to be supported by their GP, in keeping with existing literature. ${ }^{43,44}$ This highlighted the importance of GP engagement in future implementation of the intervention.

Stakeholders strongly supported medication regimen simplification for older adults receiving community-based home care services. Acceptability of the intervention was further demonstrated by the $50 \%$ implementation rate for simplification recommendations made by the clinical pharmacist. Although not specific to simplification, this is similar to implementation rates of $52-56 \%$ following medication management reviews for community dwelling people in Australia and Sweden. ${ }^{37,45}$ Those who did not opt to change their regimen still felt the service was beneficial, demonstrating the importance of shared decision-making and patient-centred care. Minor adaptions were suggested when considering a future roll-out of the intervention. For example, the need for streamlined referral systems to ensure continuity of care during future delivery of the intervention was discussed. ${ }^{46}$ The research nurse, community nurse, community-based home care provider R\&D representatives and clinical pharmacist suggested integration of a pharmacist into community-based home care teams could improve coordination, communication and medication management. The lack of easily accessible, comprehensive and accurate shared health information was also identified as a barrier to wider implementation. Many of these issues could be partly addressed through use of shared electronic healthcare systems that are currently being implemented in Australia to aid communication and continuity of care. $^{47}$

Although not designed to demonstrate effectiveness, no significant differences in the number of medication administration times or adherence were observed at follow-up. This is in contrast to a recent RACF RCT where application of MRS GRACE showed a significant reduction in the number of administration times at 4-months, that was sustained at 12month follow-up. ${ }^{23,48}$ This may be due to small sample size or 
characteristics of those recruited in the present study. The majority of participants self-managed medications and scores for the SEAMS and DRUGS questionnaires were generally high, which suggests only motivated individuals and/or those with greater health literacy may have participated, despite the older age of the cohort and high level of polypharmacy and frailty suggesting this was a high-risk cohort. Therefore, study results would still be of relevance if implemented more broadly.

\section{Strengths and Limitations}

The main limitation of this study related to recruitment challenges, which resulted in a reduction in the target sample size from 50 to 25 participants as only $12 \%$ of those invited agreed to participate. Participants were recruited from a cohort receiving community-based home care services from one service provider which may limit generalizability of the study findings.

A strength of the study was the use of a mixed-methods approach to assess feasibility. The pharmacist used a validated tool (MRS GRACE) to identify opportunities to simplify medication regimens. The clinical pharmacist drew on clinical expertise when conducting medication reconciliation however use of a validated tool to support a standardized approach for medication reconciliation should be considered in a future RCT. We did not collect information on the frequency of contact with GPs or community pharmacists during the study period.

Another limitation of this study is the inability to test a randomization method for application in a subsequent RCT because all participants received the intervention. Time spent by the single, experienced clinical pharmacist delivering the intervention was reported, however may not be representative of time spent by all pharmacists. In addition, time spent by community-based home care provider staff, GPs and community pharmacists who participated in the process was not measured.

Limitations relating to the qualitative component of the study include the small number of interview participants. Although six individuals who received the intervention were randomly selected to be interviewed, it is possible that qualitative findings may have been influenced by the method used to sample these and other interview participants. As there were no GPs interviewed, acceptability to GPs is unknown.

\section{Conclusion}

The multicomponent medication management intervention tested in this study was found to be feasible for future implementation. The high degree of intervention acceptability suggests that the 5-step regimen simplification process was well suited for use among recipients of communitybased home care services. However, barriers to recruitment were identified that should be addressed prior to progressing to an RCT or implementing the intervention into routine clinical practice. Lower than expected recruitment identified the need for an increased focus on promotional opportunities and enablers. Although the intervention components and questionnaires administered were generally well received, alterations to reduce the complexity and repetition of questions when gathering participant information are recommended. Future interventions comprising medication reconciliation, assessment of self-capacity to administer medications and medication simplification will need to ensure increased communication and collaboration between health care providers, particularly GPs, to support recruitment and implementation of recommendations.

\section{Abbreviations}

ADL, activities of daily living; BPMH, best possible medication history; CDPC, Cognitive Decline Partnership Centre; DRUGS, Drug Regimen Unassisted Grading Scale; DSRS, Dementia Severity Rating Scale; ED, emergency department; GP, general medical practitioner; IQR, interquartile range; MRS GRACE, Medication Regimen Simplification Guide for Residential Aged CarE; NHMRC, National Health and Medical Research Council; QoL-AD, Quality of Life in Alzheimer's Disease scale; RACFs, residential aged care facilities; RCT, randomized controlled trial; R\&D, research and development; SAPS, Short Assessment of Patient Satisfaction; SD, standard deviation; SEAMS, Self-Efficacy for Appropriate Medication use Scale; US, United States.

\section{Data Sharing Statement}

Requests for results of additional analyses from this pilot and feasibility study should be directed to the corresponding author.

\section{Funding}

This work was supported by the National Health and Medical Research Council (NHMRC) Partnership Centre: Dealing with Cognitive and Related Functional Decline in Older People (known as the Cognitive Decline Partnership Centre (CDPC)) (grant number GNT9100000). The CDPC received support from the NHMRC and funding partners including Helping Hand Aged Care, Hammond Care, 
Brightwater and Dementia Australia. The contents of the published materials are solely the responsibility of the individual authors identified and do not reflect the views of the NHMRC and any other funding bodies or the funding partners.

\section{Disclosure}

This study was funded through the NHMRC CDPC and the community-based home care provider organization involved in this research is one of the NHMRC CDPC funding partners. The authors report grants from the National Health and Medical Research Council Cognitive Decline Partnership Centre, during the conduct of the study. JKS is supported by an NHMRC Early Career Fellowship. JSB is supported by an NHMRC Dementia Leadership Fellowship. MC, JVE, MH and TC are employed by the organization providing the community-based home care services.

\section{References}

1. Charlesworth CJ, Smit E, Lee DSH, Alramadhan F, Odden MC. Polypharmacy among adults aged 65 years and older in the United States: 1988-2010. J Gerontol a Biol Sci Med Sci. 2015;70 (8):989-995. doi:10.1093/gerona/glv013

2. Gnjidic D, Hilmer SN, Blyth FM, et al. High-risk prescribing and incidence of frailty among older community-dwelling men. Clin Pharmacol Ther. 2012;91(3):521-528. doi:10.1038/clpt.2011.258

3. Sluggett JK, Stasinopoulos J, Bell JS. Medication management by informal caregivers - under-recognized and under-supported. Res Social Adm Pharm. 2018;14(3):215-217. doi:10.1016/j.sapharm.2017.11.002

4. Elliott RA, Goeman D, Beanland C, Koch S. Ability of older people with dementia or cognitive impairment to manage medicine regimens: a narrative review. Curr Clin Pharmacol. 2015;10 (3):213-221. doi:10.2174/1574884710666150812141525

5. Wenger N, Doherty C, Gurwitz J, et al. Optimization of drug prescription and medication management in older adults with cardiovascular disease. Drugs Aging. 2017;34(11):803-810. doi:10.1007/s40266-017-0494-2

6. Kuo SZ, Haftek M, Lai JC. Factors associated with medication non-adherence in patients with end-stage liver disease. Dig Dis Sci. 2017;62(2):543-549. doi:10.1007/s10620-016-4391-z

7. Maddigan SL, Farris KB, Keating N, Wiens CA, Johnson JA. Predictors of older adults' capacity for medication management in a self-medication program: a retrospective chart review. J Aging Health. 2003;15(2):332-352. doi:10.1177/0898264303251893

8. Onder G, Vetrano DL, Marengoni A, Bell JS, Johnell K, Palmer K. Accounting for frailty when treating chronic diseases. Eur J Intern Med. 2018;56:49-52. doi:10.1016/j.ejim.2018.02.021

9. Pantuzza LL, Ceccato M, Silveira MR, Junqueira LMR, Reis AMM. Association between medication regimen complexity and pharmacotherapy adherence: a systematic review. Eur J Clin Pharmacol. 2017;73(11):1475-1489. doi:10.1007/s00228-017-2315-2

10. Lalic S, Sluggett JK, Ilomäki J, et al. Polypharmacy and medication regimen complexity as risk factors for hospitalization among residents of long-term care facilities: a prospective cohort study. J Am Med Dir Assoc. 2016;17(11):1067.e1-.e6. doi:10.1016/j.jamda.2016.08.019

11. Wimmer BC, Cross AJ, Jokanovic N, et al. Clinical outcomes associated with medication regimen complexity in older people: a systematic review. J Am Geriatr Soc. 2017;65(4):747-753. doi:10.1111/jgs. 14682
12. Skelton F, Kunik ME, Regev T, Naik AD. Determining if an older adult can make and execute decisions to live safely at home: a capacity assessment and intervention model. Arch Gerontol Geriatr. 2010;50(3):300-305. doi:10.1016/j.archger.2009.04.016

13. The Registry of Older South Australians (ROSA). ROSA Stage 1 Historical Cohort Report [Homepage on the Internet]. Adelaide: South Australian Health and Medical Research Institute; 2018. Available from: https://rosaresearch.org/reports. Accessed February 10, 2020.

14. Giovannetti ER, Wolff JL, Xue Q-L, et al. Difficulty assisting with health care tasks among caregivers of multimorbid older adults. J Gen Intern Med. 2012;27(1):37-44. doi:10.1007/s11606-011-1831-5

15. Mira JJ, Lorenzo S, Guilabert M, Navarro I, Pérez-Jover V. A systematic review of patient medication error on self-administering medication at home. Expert Opin Drug Saf. 2015;14(6):815-838. doi:10.1517/ 14740338.2015.1026326

16. Parand A, Garfield S, Vincent C, Franklin BD. Carers' medication administration errors in the domiciliary setting: a systematic review. PLoS One. 2016;11(12):e0167204. doi:10.1371/journal.pone.0167 204

17. Elliott RA, Lee CY, Beanland C, Vakil K, Goeman D. Medicines management, medication errors and adverse medication events in older people referred to a community nursing service: a retrospective observational study. Drugs Real World Outcomes. 2016;3(1):13-24. doi:10.1007/s40801-016-0065-6

18. Chen EY, Sluggett JK, Ilomäki J, et al. Development and validation of the Medication Regimen Simplification Guide for Residential Aged CarE (MRS GRACE). Clin Interv Aging. 2018;13:975-986. doi:10.2147/CIA.S158417

19. Flynn AJ, Klasnja P, Friedman CP. MedMinify: an advice-giving system for simplifying the schedules of daily home medication regimens used to treat chronic conditions. AMIA Annu Symp Proc. 2014;2014:1728-1737.

20. Jung SH, Lee OS, Kim HS, et al. Medication adherence improvement by using Administration Timing Simplification Protocol (ATSP) in cardiovascular disease patients. J Atheroscler Thromb. 2017;24 (8):841-852. doi:10.5551/jat.36335

21. Sluggett JK, Chen EYH, Ilomäki J, et al. SImplification of Medications Prescribed to Long-tErm care Residents (SIMPLER): study protocol for a cluster randomized controlled trial. Trials. 2018;19(1):37. doi:10.1186/s13063-017-2417-2

22. Chen EYH, Bell JS, Ilomäki J, et al. Medication regimen complexity in 8 Australian residential aged care facilities: impact of age, length of stay, comorbidity, frailty, and dependence in activities of daily living. Clin Interv Aging. 2019;14:1783-1795. doi:10.2147/CIA. S216705

23. Sluggett JK, Chen EYH, Ilomäki J, et al. Reducing the burden of complex medication regimens: Simplification of Medications Prescribed to Long-tErm care Residents (SIMPLER) cluster randomized controlled trial. J Am Med Dir Assoc. 2020. doi:10.1016/j. jamda.2020.02.003

24. Sluggett JK, Page AT, Chen EYH, et al. Protocol for a non-randomized pilot and feasibility study evaluating a multicomponent intervention to simplify medication regimens for people receiving community-based home care services. BMJ Open. 2019;9:e025345. doi:10.1136/bmjopen-2018-025345

25. Commonwealth Home Support Programme [homepage on the Internet]. Australian Government; 2020. Available from: https:// www.myagedcare.gov.au/help-home/commonwealth-home-supportprogramme. Accessed February 8, 2020.

26. Home Care Packages [homepage on the Internet]. Australian government; 2020. Available from: https://www.myagedcare.gov.au/ help-at-home/home-care-packages. Accessed February 8, 2020.

27. Clark C, Ewbank D. Performance of the dementia severity rating scale: a caregiver questionnaire for rating severity in Alzheimer disease. Alzheimer Dis Assoc Disord. 1996;10(1):31-39. 
28. Katz S, Ford AB, Moskowitz RW, Jackson BA, Jaffe MW. Studies of illness in the aged: the index of ADL: a standardized measure of biological and psychosocial function. JAMA. 1963;185(12):914-919. doi:10.1001/jama.1963.03060120024016

29. Morley JE, Malmstrom TK, Miller DK. A simple frailty questionnaire (FRAIL) predicts outcomes in middle aged African Americans. $J$ Nutr Health Aging. 2012;16(7):601-608. doi:10.1007/s12603-0120084-2

30. Edelberg HK, Shallenberger E, Wei JY. Medication management capacity in highly functioning community-living older adults: detection of early deficits. J Am Geriatr Soc. 1999;47(5):592-596. doi:10.1111/j.1532-5415.1999.tb02574.x

31. Braun V, Clarke V. Thematic analysis: a reflexive approach [homepage on the internet]. The University of Auckland; 2020. Available from: https://www.psych.auckland.ac.nz/en/about/thematic-analysis. html. Accessed April 17, 2020.

32. Jessica R, Jacobson TA, Sunil F. Development and psychometric evaluation of the Self-Efficacy for Appropriate Medication Use Scale (SEAMS) in low-literacy patients with chronic disease J Nurs Meas. 2007;15(3):203-219. doi:10.1891/106137407783095 757

33. Logsdon RG, Gibbons LE, McCurry SM, Teri L. Quality of life in alzheimer's disease: patient and caregiver reports. J Ment Health Aging. 1999;5(1):21-32.

34. Hawthorne G, Sansoni J, Hayes L, Marosszeky N, Sansoni E. Measuring patient satisfaction with health care treatment using the short assessment of patient satisfaction measure delivered superior and robust satisfaction estimates. $J$ Clin Epidemiol. 2014;67 (5):527-537. doi:10.1016/j.jclinepi.2013.12.010

35. Claeys C, Neve J, Tulkens P, Spinewine A. Content validity and inter-rater reliability of an instrument to characterize unintentional medication discrepancies. Drugs Aging. 2012;29(7):577-591. doi:10. 2165/11631980-000000000-00000

36. Wong JD, Bajcar JM, Wong GG, et al. Medication reconciliation at hospital discharge: evaluating discrepancies. Ann Pharmacother. 2008;42(10):1373-1379. doi:10.1345/aph.1L190

37. Lee CY, Beanland C, Goeman D, et al. Improving medication safety for home nursing clients: a prospective observational study of a novel clinical pharmacy service - the Visiting Pharmacist (ViP) study. J Clin Pharm Ther. 2018;43(6):813-821. doi:10.1111/jcpt.12712

38. Twigg MJ, Bhattacharya D, Desborough JA, Wright D. A drop-in clinic for patients with poorly-controlled diabetes: a community pharmacy feasibility study. Int $J$ Clin Pharm. 2015;37(2):395-402. doi:10.1007/s11096-015-0076-5
39. Kwint HF, Faber A, Gussekloo J, Bouvy ML. The contribution of patient interviews to the identification of drug-related problems in home medication review. J Clin Pharm Ther. 2012;37(6):674-680. doi:10.1111/j.1365-2710.2012.01370.x

40. Verdoorn S, Kwint H-F, Blom JW, Gussekloo J, Bouvy ML. Effects of a clinical medication review focused on personal goals, quality of life, and health problems in older persons with polypharmacy: a randomized controlled trial (DREAMeR-study). PLoS Med. 2019;16(5):e1002798. doi:10.1371/journal.pmed.1002798

41. Carter SR, Moles R, White L, Chen TF. Patients' willingness to use a pharmacist-provided medication management service: the influence of outcome expectancies and communication efficacy. Res Social Adm Pharm. 2012;8(6):487-498. doi:10.1016/j.sapharm.2012.01.002

42. Lee CY, George J, Elliott RA, Stewart K. Exploring stakeholder perspectives on medication review services for older residents in retirement villages. Int J Pharm Pract. 2012;20(4):249-258. doi:10. 1111/j.2042-7174.2012.00189.x

43. Carter SR, Moles RJ, White L, Chen TF. Consumers' willingness to use a medication management service: the effect of medicationrelated worry and the social influence of the general practitioner. Res Social Adm Pharm. 2013;9(4):431-445. doi:10.1016/j.sapharm. 2012.07.001

44. Kalogianis MJ, Wimmer BC, Turner JP, et al. Are residents of aged care facilities willing to have their medications deprescribed? Res Social Adm Pharm. 2016;12(5):784-788. doi:10.1016/j.sapharm.20 15.12.004

45. Milos V, Rekman E, CBondesson A, et al. Improving the quality of pharmacotherapy in elderly primary care patients through medication reviews: a randomized controlled study. Drugs Aging. 2013;30: 235-246. doi:10.1007/s40266-013-0057-0

46. Twigg M, Wright D, Kirkdale C, Desborough J, Thornley T, O'Connor B. The UK pharmacy care plan service: description, recruitment and initial views on a new community pharmacy intervention. PLoS One. 2017;12(4):e0174500. doi:10.1371/journal. pone. 0174500

47. Australian Digital Health Agency. My Health Record [Homepage on the Internet]. Australian Government: Australian Digital Health Agency; 2019. Available from https://www.myhealthrecord.gov.au/. Accessed January 20, 2020.

48. Sluggett JK, Chen EYH, Ilomäki J, et al. Impact of medication regimen simplification on medication administration times and health outcomes in residential aged care: 12 month follow up of the SIMPLER randomized controlled trial. J Clin Med. 2020;9(4): E1053. doi:10.3390/jcm9041053
Clinical Interventions in Aging

\section{Publish your work in this journal}

Clinical Interventions in Aging is an international, peer-reviewed journal focusing on evidence-based reports on the value or lack thereof of treatments intended to prevent or delay the onset of maladaptive correlates of aging in human beings. This journal is indexed on PubMed Central, MedLine, CAS, Scopus and the Elsevie
Bibliographic databases. The manuscript management system is completely online and includes a very quick and fair peer-review system, which is all easy to use. Visit http://www.dovepress.com/ testimonials.php to read real quotes from published authors. 